\title{
Release of yeast telomeres from the nuclear periphery is triggered by replication and maintained by suppression of Ku-mediated anchoring
}

\author{
Hani Ebrahimi and Anne D. Donaldson ${ }^{1}$ \\ Institute of Medical Sciences, University of Aberdeen, Foresterhill, Aberdeen AB25 2ZD, Scotland, United Kingdom
}

\begin{abstract}
The perinuclear localization of Saccharomyces cerevisiae telomeres provides a useful model for studying mechanisms that control chromosome positioning. Telomeres tend to be localized at the nuclear periphery during early interphase, but following $\mathrm{S}$ phase they delocalize and remain randomly positioned within the nucleus. We investigated whether DNA replication causes telomere delocalization from the nuclear periphery. Using live-cell fluorescence microscopy, we show that delaying DNA replication causes a corresponding delay in the dislodgment of telomeres from the nuclear envelope, demonstrating that replication of individual telomeres causes their delocalization. Telomere delocalization is not simply the result of recruitment to a replication factory in the nuclear interior, since we found that telomeric DNA replication can occur either at the nuclear periphery or in the nuclear interior. The telomere-binding complex Ku is one of the factors that localizes telomeres to the nuclear envelope. Using a gene locus tethering assay, we show that Ku-mediated peripheral positioning is switched off after DNA replication. Based on these findings, we propose that DNA replication causes telomere delocalization by triggering stable repression of the Ku-mediated anchoring pathway. In addition to maintaining genetic information, DNA replication may therefore regulate subnuclear organization of chromatin.
\end{abstract}

[Keywords: Telomere; replication; nuclear organization; Saccharomyces cerevisiae]

Supplemental material is available at http://www.genesdev.org.

Received May 6, 2008; revised version accepted September 29, 2008.

Eukaryotic chromosomes are nonrandomly positioned within the nucleus, and there is substantial evidence for the functional importance of chromosome positioning. Changes in the subnuclear organization of chromosomes are thought to facilitate both the regulation of gene expression and productive interactions between genetic loci (Gottschling et al. 1990; Aparicio et al. 1991; Cremer and Cremer 2001). For example, the recombination and transcriptional states of immunoglobulin genes during $B$ lymphocyte development appear to be regulated by the location of these loci at or away from the nuclear periphery (Kosak et al. 2002). Perinuclear positioning of genes has also been implicated in transcriptional control in Saccharomyces cerevisiae. Proteins involved in transcriptional silencing, such as Sir4p, are enriched in discrete regions at the nuclear periphery (Maillet et al. 1996; Cockell and Gasser 1999), and positioning of chromosomal loci within these regions appears to facilitate tran-

${ }^{1}$ Corresponding author.

E-MAIL a.d.donaldson@abdn.ac.uk; FAX 44-1224-555844.

Article is online at http://www.genesdev.org/cgi/doi/10.1101/gad.486208. scription repression (Andrulis et al. 1998). The S. cerevisiae mating-type loci and telomeres (chromosomes ends) both constitute regions of silent chromatin that tend to localize in the vicinity of the nuclear envelope (Gottschling et al. 1990; Aparicio et al. 1991; Gotta et al. 1996; Hediger et al. 2002). Association of a gene locus with the nuclear envelope does not, however, necessarily lead to transcriptional repression, and indeed can conversely promote gene expression (Ishii et al. 2002; Brickner and Walter 2004; Casolari et al. 2005; Taddei et al. 2006). On the basis of these observations, the nuclear periphery has been proposed to consist of a mosaic of microenvironments, some of which favor silencing and others transcriptional activation (Taddei et al. 2006; Kumaran et al. 2008; Meaburn and Misteli 2008). In yeast the perinuclear localization of telomeres is important not only to reinforce telomeric silencing but also for efficient repair of double-strand breaks at subtelomeric regions (Therizols et al. 2006), suggesting that three-dimensional organization of chromatin affects multiple biochemical processes.

Telomeres are conserved nucleoprotein structures that form the natural ends of linear chromosomes. They per- 
form multiple functions including end protection to ensure genome integrity (Louis and Vershinin 2005) and recruitment of telomerase to maintain telomere length through successive rounds of DNA replication. Sir4p and the heterodimeric protein complex $\mathrm{Ku}$ /consisting of $\mathrm{Ku} 70$ and $\mathrm{Ku} 80$ ) are among many telomeric proteins that bind to telomere sequences throughout the yeast cell cycle. Sir4 and $\mathrm{Ku}$ function in telomere length maintenance, in establishment of silencing at subtelomeric regions (Gotta et al. 1996; Fisher and Zakian 2005), and in intranuclear positioning of telomeres (Taddei and Gasser 2004). The subnuclear localization of telomeres has been explored in various species (Funabiki et al. 1993; Postberg et al. 2001; Schmitt et al. 2007), but the molecular pathways that function in telomere positioning are best understood in budding yeast. In this organism, localization of telomeres is maintained via two different pathways that anchor telomeres to the nuclear envelope. Sir4p and $\mathrm{Ku}$ complex characterize the two telomereanchoring pathways, and ablating both pathways causes delocalization of all telomeres examined (Palladino et al. 1993; Laroche et al. 1998; Hediger et al. 2002). Localization of different telomeres shows variable sensitivity to loss of either $\mathrm{Ku}$ or Sir4 function, implying that certain telomeres are primarily anchored via the Sir4 pathway, while others depend more on the anchoring function of $\mathrm{Ku}$. Sir4 is believed to establish telomere anchoring by interacting with the peripherally localized protein Esc1 and nuclear pore proteins (Taddei et al. 2004; Therizols et al. 2006). Esclp also contributes to Ku-mediated anchoring; however, an unidentified Esc1-independent factor is additionally involved in anchoring via the Ku pathway (Taddei et al. 2004). As well as Ku and Sir4, recent studies have identified the proteins Ctf18 and Mps3 as required for localization of telomeres at the nuclear periphery (Hiraga et al. 2006; Bupp et al. 2007).

The dynamics of telomere positioning in live $S$. cerevisiae cells have been studied, both in terms of shortterm mobility and longer-term cell cycle-regulated localization. Early in the cell cycle (prior to bud emergence), telomeres tend to localize at the nuclear periphery. In large-budded cells, telomeres are dislodged from the nuclear periphery and remain randomly positioned as cells prepare for mitosis (Laroche et al. 2000; Hediger et al. 2002). The cell cycle mechanism that alters telomere localization in late interphase has not previously been characterized. Interestingly, however, it has been suggested that reorganization of telomeres following premeiotic $S$ phase may be linked to DNA replication (Trelles-Sticken et al. 2005).

Even during the early part of the cell cycle when telomeres are peripherally positioned, anchoring of telomeres to the nuclear envelope is dynamic. Real-time observation of a GFP-tagged locus at a chromosome end reveals substantial short-term mobility in unbudded cells, with periods in which a particular telomere is peripherally localized interspersed by intervals of apparently random diffusive movement within the nuclear interior (Heun et al. 2001b; Hiraga et al. 2006). This saltatory mobility means that telomeres are not observed to be at the nuclear periphery in all unbudded cells-for instance, the right telomere of chromosome VI is seen localized to the periphery in $\sim 60 \%$ of unbudded cells, while the left telomere of chromosome VIII is found at the nuclear periphery in $\sim 50 \%$ (Hediger et al. 2002). These levels of positioning within a population are similar to those displayed by other $S$. cerevisiae genetic loci that undergo peripheral localization (Brickner et al. 2007).

Since telomere delocalization during mitotic interphase approximately coincides with DNA replication, we investigated the effect of replication on telomere anchoring to the nuclear envelope. By manipulating the replication kinetics of the entire genome or of a specific telomere, we show that telomere delocalization depends directly on DNA replication. To maintain telomere delocalization, the peripheral anchoring pathways must presumably be rendered ineffective, so we examined the effect of replication on the peripheral-anchoring functions of Ku80 and Sir4 proteins. We found that following DNA replication, the Ku80-mediated anchoring pathway is switched off, suggesting the likely cause of the observed delocalization of telomeres from the nuclear periphery in late interphase.

\section{Results}

Timely telomere dislodgement is dependent on expression of $S$-phase cyclins

We wished to examine whether dislodgment of telomeres from the nuclear envelope depends on DNA replication. Our first approach was to manipulate the cell cycle controls over DNA replication so that the onset of $S$ phase was delayed in relation to other cell cycle events, and to test the effect on telomere localization. Cell cycle events in S. cerevisiae depend on Cdc28 protein kinase. Throughout the cell cycle, various cyclins activate Cdc28 and determine its function. Two B-type cyclins, Clb5 and Clb6, drive the timely initiation of chromosome replication (Epstein and Cross 1992; Kuhne and Linder 1993). Deleting CLB5 and CLB6 delays replication initiation until expression of mitotic cyclins is switched on; hence, a clb5 clb6 mutant displays a delay of $\sim 25 \mathrm{~min}$ in entering $\mathrm{S}$ phase after release from $\alpha$-factor (Fig. 1A; Schwob and Nasmyth 1993). Bud emergence and growth are not delayed by clb5 clb6 deletion (Fig. 1B; Supplemental Fig. S1) as expected, since budding depends on the G1 cyclins (CLNs).

If replication causes telomeres to dislodge from the nuclear periphery, then in clb5 clb6 cells, the dislodgment of telomeres is expected to be delayed because of the delayed replication. To study the positioning of telomeres in live cells, telomeric loci can be tagged with GFP using the "lac operator/repressor recognition" system (Robinett et al. 1996). In this method, an array of lac operator repeats $\left(\mathrm{lac}^{\mathrm{OP}}\right)$ is inserted close to a telomere, in cells expressing the Lac repressor protein fused to GFP (LacI-GFP). Recruitment of LacI-GFP to the tagged telo- 
mere allows its visualization as a bright globular dot using fluorescence microscopy (Fig. 1C). To determine the position of the fluorescent telomeric dot within the nucleus, the spherical boundary of the nucleus is visualized using nuclear pore component Nup49 fused to GFP (Heun et al. 2001b). Figure 1C illustrates the previously established "nuclear zoning assay" for quantifying telomere localization (Hediger et al. 2002), used to examine whether a telomere is positioned at the nuclear envelope. A randomly positioned telomere will be equally distributed between three zones of equal area ( $33 \%$ in Zones I, II, and III), whereas a peripherally localized telomere will be preferentially positioned in Zone I. In the graphs showing telomere position data in Figure 1, D and E, the horizontal dashed line at 33\% corresponds to a random distribution.

We studied localization of telomere VIII-left (Tel VIIIleft) at different stages of the cell cycle assessed on the basis of bud size. In unbudded and small-budded wildtype cells (early interphase), Tel VIII-left showed significant enrichment in the peripheral Zone I (Fig. 1D, black bars). However, in medium- and large-budded cells (late interphase), Tel VIII-left was distributed randomly within the nucleus (statistical significance values for Tel VIII-left localization are shown in Table 1). These observations were consistent with previous studies showing

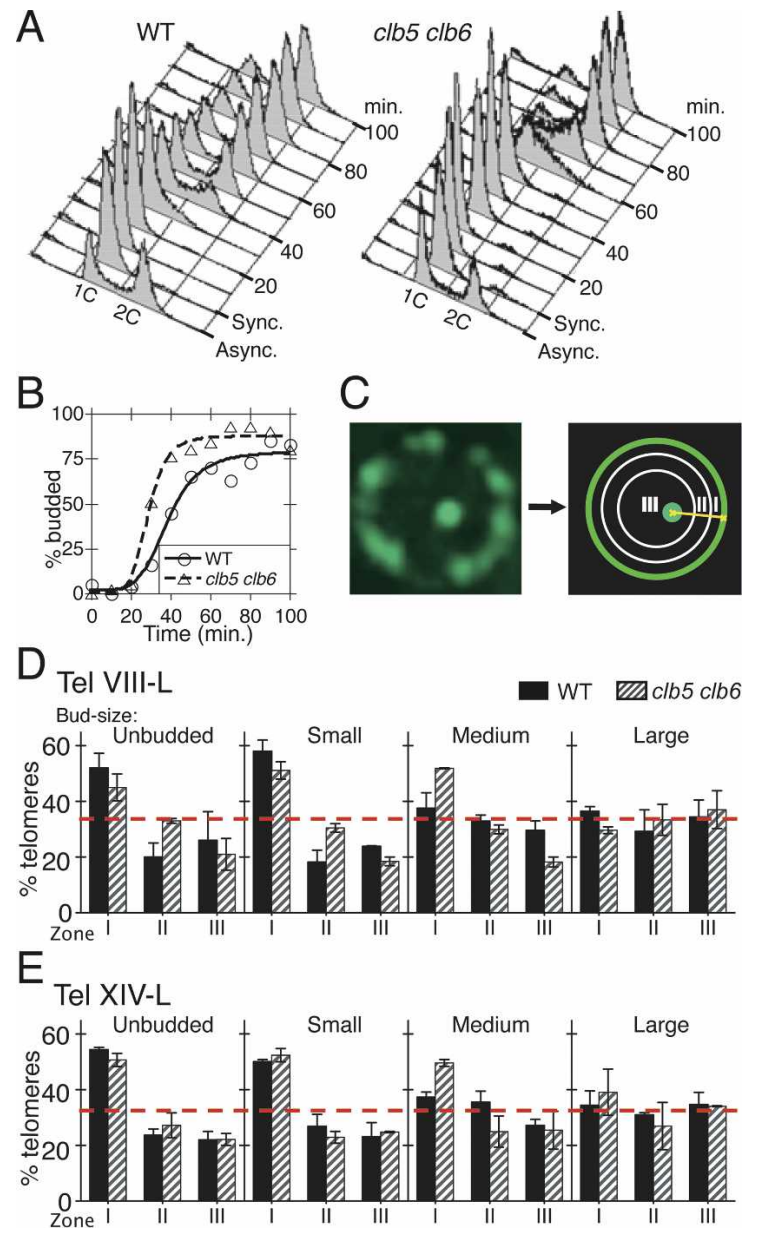

that localization of this telomere depends on cell cycle stage (Laroche et al. 2000; Hediger et al. 2002).

In clb5 clb6 cells, where onset of S phase was delayed, Tel VIII-left was localized at the nuclear periphery in unbudded and small-budded cells at levels similar to wild type (Fig. 1D, hatched bars; Table 1). However, Tel VIII-left remained significantly peripheral in mediumbudded cells, only delocalizing later in the cell cycle when cells became large-budded. Therefore, delaying DNA replication led to prolonged anchoring of Tel VIIIleft during the cell cycle.

A similar experiment was carried out examining localization of Tel XIV-left (Fig. 1E). In wild-type cells, Tel XIV-left showed a cell cycle-controlled localization pattern similar to that of Tel VIII-left-that is, delocalized in medium-budded cells. In clb5 clb6 cells, localization of Tel XIV-left was prolonged, so that it became delocalized only later in large-budded cells. Prolonged telomere localization was also observed in a clb5 clb6 strain for Telomeres VII-left and V-right (Supplemental Fig. S2). Together these data confirm that $S$. cerevisiae telomeres become delocalized in late interphase and reveal that the normal time of delocalization depends on expression of the S-phase-promoting cyclins Clb5 and Clb6.

To confirm the effect of the clb5 clb6 mutation on telomere dynamics, we examined intranuclear foci formed by the telomeric marker protein Rap1-GFP (Hayashi et al. 1998). Disengagement of Rapl foci from the nuclear periphery was delayed in clb5 clb6 when

Figure 1. Delocalization of telomeres VIII-left and XIV-left is dependent on expression of S-phase cyclins. (A) To determine the time of S-phase onset, DNA content was measured by FACS analysis in synchronized cultures of wild-type (GA-1985) and clb5 clb6 strains (HE4). Samples were taken every $10 \mathrm{~min}$ after release from $\alpha$-factor synchronization. (B) Percentage of budded cells was analyzed in the synchronized cultures used in $(A) .(C$, left panel) Microscopic image showing Tel VIII-left as a bright globular dot within the fluorescent nuclear rim. (Right panel) To quantify subnuclear positioning of a telomere, the area of the nucleus is measured and equally divided into three zones (Zone I, Zone II, and Zone III). Therefore, each zone occupies one-third of the area within the nuclear rim. In each cell, the telomere dot can be assigned to one of the three zones by measuring the distance from center of the dot to the periphery (indicated by the yellow line); a telomere is considered peripheral if it is located in Zone I. In a population of cells, a randomly positioned telomere will be present in Zones I, II, and III with equal frequency $(\sim 33 \%)$, while an anchored telomere will be significantly enriched in Zone I. $(D, E)$ Bar charts show the percentage of telomeres VIII-left and XIV-left in Zone I, II, or III. The asynchronous-growing cells were divided into four categories according to their bud size: unbudded, small-budded $(<2 \mu \mathrm{m})$, mediumbudded $(2-2.75 \mu \mathrm{m})$, and large-budded $(2.75-3.5 \mu \mathrm{m})$. The horizontal dashed line at $33 \%$ indicates the expected distribution of a randomly positioned telomere. Telomere positioning was observed and quantified in two independent strain isolates (error bars show SD). To determine statistical significance of telomere localization, $P$ values from the $\chi^{2}$ test were calculated by comparing the observed numbers in each zone with the expected random distribution in each population (significance values are indicated in Table 1). 
Table 1. Statistical significance of telomere localization data in Figures 1 and 2

\begin{tabular}{|c|c|c|c|c|}
\hline $\begin{array}{l}\text { Tagged telomere } \\
\text { Strain }\end{array}$ & Unbudded & Small-budded & Medium-budded & Large-budded \\
\hline $\begin{array}{l}\text { Tel VIII-left } \\
\text { Wild type }\end{array}$ & $\begin{aligned} n_{1} & =66, n_{2}=51 \\
P & =8.42 \times 10^{-7}\end{aligned}$ & $\begin{aligned} n_{1} & =89, n_{2}=79 \\
P & =6.38 \times 10^{-5}\end{aligned}$ & $\begin{aligned} n_{1} & =97, n_{2}=65 \\
P & =9.22 \times 10^{-1}\end{aligned}$ & $\begin{aligned} n_{1} & =46, n_{2}=42 \\
P & =5.34 \times 10^{-1}\end{aligned}$ \\
\hline $\begin{array}{l}\text { Tel VIII-left } \\
\text { clb5 clb6 }\end{array}$ & $\begin{aligned} n_{1} & =56, n_{2}=62 \\
P & =1.61 \times 10^{-4}\end{aligned}$ & $\begin{aligned} n_{1} & =25, n_{2}=83 \\
P & =2.52 \times 10^{-3}\end{aligned}$ & $\begin{aligned} n_{1} & =60, n_{2}=73 \\
P & =2.83 \times 10^{-4}\end{aligned}$ & $\begin{aligned} n_{1} & =41, n_{2}=40 \\
P & =4.55 \times 10^{-1}\end{aligned}$ \\
\hline $\begin{array}{l}\text { Tel XIV-left } \\
\text { Wild type }\end{array}$ & $\begin{aligned} n_{1} & =58, n_{2}=56 \\
P & =1.36 \times 10^{-5}\end{aligned}$ & $\begin{aligned} n_{1} & =61, n_{2}=71 \\
P & =2.59 \times 10^{-4}\end{aligned}$ & $\begin{aligned} n_{1} & =92, n_{2}=76 \\
P & =4.47 \times 10^{-1}\end{aligned}$ & $\begin{aligned} n_{1} & =43, n_{2}=22 \\
P & =4.15 \times 10^{-1}\end{aligned}$ \\
\hline $\begin{array}{l}\text { Tel XIV-left } \\
\text { clb5 clb6 }\end{array}$ & $\begin{aligned} n_{1} & =60, n_{2}=66 \\
P & =2.13 \times 10^{-3}\end{aligned}$ & $\begin{aligned} n_{1} & =53, n_{2}=48 \\
P & =2.74 \times 10^{-5}\end{aligned}$ & $\begin{aligned} n_{1} & =59, n_{2}=62 \\
P & =3.21 \times 10^{-4}\end{aligned}$ & $\begin{aligned} n_{1} & =65, n_{2}=38 \\
P & =8.41 \times 10^{-1}\end{aligned}$ \\
\hline $\begin{array}{l}\text { Tel VI-right } \\
\text { Wild type }\end{array}$ & $\begin{aligned} n_{1} & =87, n_{2}=54 \\
P & =5.32 \times 10^{-16}\end{aligned}$ & $\begin{aligned} n_{1} & =93, n_{2}=75 \\
P & =1.64 \times 10^{-20}\end{aligned}$ & $\begin{aligned} n_{1} & =110, n_{2}=58 \\
P & =6.09 \times 10^{-2}\end{aligned}$ & $\begin{aligned} n_{1} & =67, n_{2}=22 \\
P & =1.08 \times 10^{-1}\end{aligned}$ \\
\hline $\begin{array}{l}\text { Tel VI-right } \\
\boldsymbol{\Delta} \mathbf{4 A R S}\end{array}$ & $\begin{aligned} n_{1} & =52, n_{2}=32 \\
P & =1.28 \times 10^{-5}\end{aligned}$ & $\begin{aligned} n_{1} & =82, n_{2}=47 \\
P & =1.08 \times 10^{-14}\end{aligned}$ & $\begin{aligned} n_{1} & =115, n_{2}=64 \\
P & =5.82 \times 10^{-12}\end{aligned}$ & $\begin{aligned} n_{1} & =67, n_{2}=52 \\
P & =1.07 \times 10^{-2}\end{aligned}$ \\
\hline
\end{tabular}

For each bud-size category, the number of observed cells from two different strain isolates is indicated by $n_{1}$ and $n_{2}$. $P$ values indicate the significance of the difference between the observed number of telomeres in Zones I, II, and III and expected random distribution.

compared to wild type (Supplemental Fig. S3), confirming the results obtained when observing single telomeres using chromosome dot strains.

\section{Delaying replication of Tel VI-right prolonged its localization at the periphery}

Our observation of telomeres in clb5 clb6 strains showed that expression of S-phase cyclins causes timely telomere dislodgment from the nuclear envelope. Clb5 and Clb6 proteins might affect telomere localization by initiating DNA replication, or through a different uncharacterized pathway such as phosphorylation of a substrate involved in anchoring. In order to investigate whether replication directly affects telomere positioning, we designed an experiment in which the replication time of a single telomere is delayed.

Eukaryotic DNA replication initiates from several replication origins dispersed along the chromosomes. In $S$. cerevisiae, replication origins are known as "autonomous replication sequences," or ARS elements, and their activity depends on a defined ARS consensus sequence (ACS). There are five replication origins on the right arm of chromosome VI (Chr. VI-R) (Friedman et al. 1997; Yamashita et al. 1997; Raghuraman et al. 2001). The telomere-proximal origin ARS610 lies within the X core subtelomeric element (STE) and is located 126 base pairs (bp) from Tel VI-right. The other four origins, called ARS609, ARS608, ARS607, and ARS606, are located respectively $13 \mathrm{~kb}, 53 \mathrm{~kb}, 70 \mathrm{~kb}$, and $102 \mathrm{~kb}$ away from Tel VI-right (Fig. 2A). Therefore, we aimed to delay the replication of Tel VI-right by deleting the four most telomere-proximal origins (ARS607, ARS608, ARS609, and ARS610) on Chr. VI-R. All four replication origins were replaced by selectable markers, creating a strain that we refer to as $\triangle 4 A R S$ (Fig. 2A). In $\triangle 4 A R S$ cells, the Tel VI-right region is expected to be replicated by a rightward-moving replication fork originating from ARS606. Since ARS606 lies $102 \mathrm{~kb}$ away from Tel VI-right, an abnormally large DNA region must be replicated by this replication fork before it arrives at Tel VI-right. The unusually late arrival of a replication fork at the Tel VI-right region is expected to cause a delay in the replication of this region.

To confirm that replication of Tel VI-right was delayed in $\triangle 4 A R S$ cells, the replication time of the right end of chromosome VI was measured using the dense isotope transfer method. In this technique, the replication time of genomic fragments is measured by monitoring the synthesis of isotopically labeled nascent DNA (McCarroll and Fangman 1988; Donaldson et al. 1998). Figure 2B shows the replication kinetics of two known early-replicating loci (ARS305 and ARS1) and a known late-replicating locus on chromosome XIV (Chr. XIV-internal). Measuring replication kinetics of Tel VI-right in the wild-type strain revealed that this region replicates rather late in S phase (Fig. 2B, left panel, filled diamonds). This result was consistent with the fact that $S$. cerevisiae telomeres generally replicate late (Ferguson et al. 1991; Raghuraman et al. 2001). Figure 2B (right panel) shows replication kinetics of the same four genomic sequences in the $\triangle 4 A R S$ strain. Deleting the ARS elements on Chr. VI-R did not alter the replication time of ARS305, ARS1, or Chr. XIV-internal, as expected since these sequences lie on chromosomes III, IV, and XIV. In wild-type cells, Tel VI-right replicated at approximately the same time as the late-replicating marker sequence (Chr. XIV-internal). However, in the $\triangle 4 A R S$ cells, replication of Tel VI-right was noticeably delayed, with replication proceeding considerably later and less synchronously than the Chr. XIV-internal marker.

In these experiments, the replication time of individual loci can be assigned as the time at which half of the final replication level has been achieved (Friedman et al. 1996). Figure 2C shows the measured replication times for the same four loci, plotted relative to the replication time of early-replicating marker ARS305 in each strain. This analysis showed that deleting the replication origins caused a delay in replication of Tel VI-right of approximately $8 \mathrm{~min}$. To summarize, the replication timing analysis showed that deleting ARS607, ARS608, ARS609, and ARS610 delayed and increased asynchrony of replication of the right end of the chromosome VI, 
A

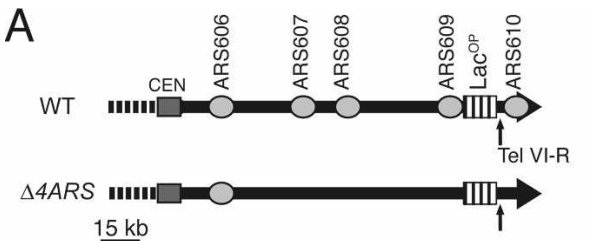

B

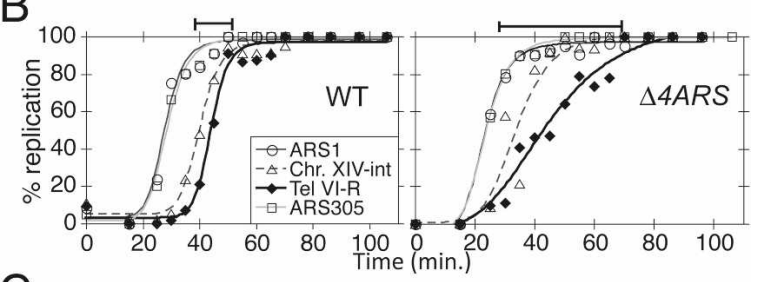

C
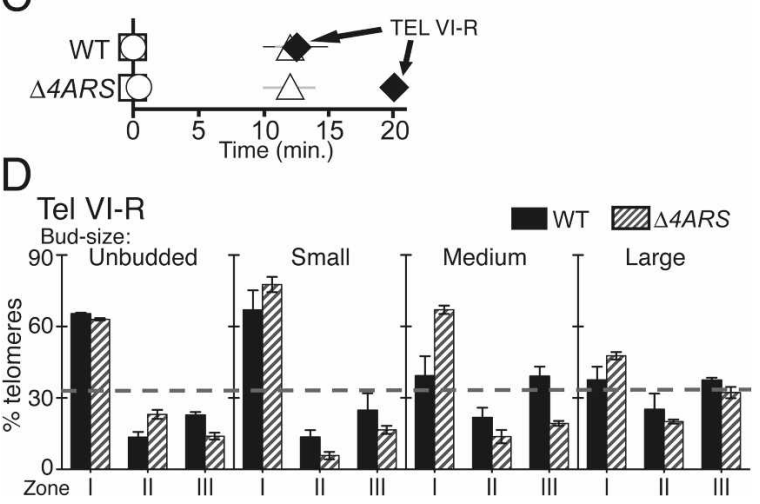

Figure 2. Delaying replication of Tel VI-right postpones its dislodgment from the nuclear periphery. (A) Schematic presentation of the right arm of chromosome VI in wild-type (GA-1459) and $\triangle 4 A R S$ (HE62) strains. Replication origins ARS606, ARS607, ARS608, ARS609, and ARS610 are shown as ovals. The inserted array of lac ${ }^{\mathrm{OP}}$ repeats at the subtelomeric region is shown as a vertically-hatched rectangle. Vertical arrows indicate the position of the DNA fragment analyzed to measure the replication time of Tel VI-right. Replication origins ARS607, ARS608, ARS609, and ARS610 are deleted in the $\triangle 4 A R S$ strain $(B)$ Plots show the percentage of cells that have replicated ARS1, ARS305, Chr. XIV-internal, and Tel VI-right fragments at time points taken after release from $\alpha$-factor. ARS1 and ARS305 are known early-replicating fragments; the chromosome XIV-internal fragment is a late-replicating marker. Horizontal lines above the graphs indicate the time taken for Tel VI-right to be replicated in the central $80 \%$ of cycling cells (a 14-min. interval for wild type, and $42 \mathrm{~min}$. for $\triangle 4 A R S$ ). The replication time of each fragment is measured as the time at which it has been replicated in half of the cycling cells. $(C)$ Replication time values of ARS1 (circles), Chr. XIV-internal (triangles), and Tel VI-right (black diamonds) plotted relative to the replication time of ARS305 (squares), which is assigned a value of $0 \mathrm{~min}$. Both dense isotope transfer experiments were repeated on independent cultures; error bars show the SD. $(D)$ Bar charts show the localization of Tel VI-right in wild-type and $\triangle 4 A R S$ cells plotted using notation similar to that in Figure 1. (For statistical significance of Tel VI-right localization, see Table 1.)

without affecting budding (Supplemental Fig. S4) or replication of other genomic regions.

Localization of Tel VI-right was studied in wild-type and $\triangle 4 A R S$ cells. In wild-type cells, the observed local- ization of Tel VI-right was in concordance with previously published data (Hediger et al. 2002) - that is, Tel VI-right was localized to the periphery in unbudded and small-budded cells but delocalized in medium and largebudded cells (Fig. 2D, black bars; Table 1). In $\triangle 4 A R S$ cells, where replication of Tel VI-right had been delayed, we found that localization of Tel VI-right at the nuclear periphery was prolonged. Tel VI-right remained significantly enriched in Zone I in medium-budded cells and only became delocalized later in large-budded cells (Fig. $2 \mathrm{D}$, hatched bars). It is notable that deleting replication origins on Chr. VI-R did not significantly affect telomere tethering in unbudded and small-budded cells, suggesting that in early interphase, telomere localization does not depend on events that occur at replication origins (such as recruitment of origin licensing proteins). The above data show that cell cycle delocalization of Tel VIright in medium-budded cells depends on timely DNA replication of this particular telomere. This observation suggests that the effect of clb5 clb6 in delaying telomere dislodgment was probably also directly due to the delay in initiation of DNA replication.

\section{Telomeres of chromosome VI are independently tethered to the nuclear envelope}

It has been shown that during interphase, the left and right telomeres of chromosome VI tend to be located in close proximity. In addition, Tel VI-right and Tel VI-left show coordinated movement within the nucleus (Bystricky et al. 2005). However, it is not known whether the peripheral localization of these juxtaposed telomeres is coordinately regulated. It is possible to observe both telomeres of chromosome VI in a single nucleus by inserting arrays of $\mathrm{lac}^{\mathrm{OP}}$ and tet ${ }^{\mathrm{OP}}$ repeats at Tel VI-right and Tel VI-left, respectively (Fig. 3A). Expressing both LacI-CFP and TetR-YFP fusion proteins in this strain enables these two telomeres to be visualized and distinguished in each live cell. Expression of TetR-YFP generates a uniform background across the nucleoplasm that can be used to visualize nuclear shape and determine the center and diameter of the nucleus (Hediger et al. 2004; Bystricky et al. 2005).

To address whether the left and right telomeres of Chr. VI are independently affected by DNA replication, we delayed replication of Tel VI-right by deleting ARS607, ARS608, ARS609, and ARS610 as described for the $\triangle 4 A R S$ strain above (Fig. 3A). As expected, in unbudded and small-budded wild-type cells, both Tel VI-right and Tel VI-left were localized to the nuclear periphery. In medium- and large-budded wild-type cells, both the left and right chromosome ends dislodged from the nuclear envelope (Fig. 3B, black bars). In $\triangle 4 A R S$ cells, where replication of Tel VI-right was delayed, localization of this telomere at the nuclear periphery was prolonged, with Tel VI-right remaining anchored to the nuclear envelope in medium-budded cells (Fig. 3B, top graphs, hatched bars). However, the prolonged anchoring of Tel VI-right did not affect localization of Tel VI-left, since this telomere dislodged at its normal time in medium-budded 
A

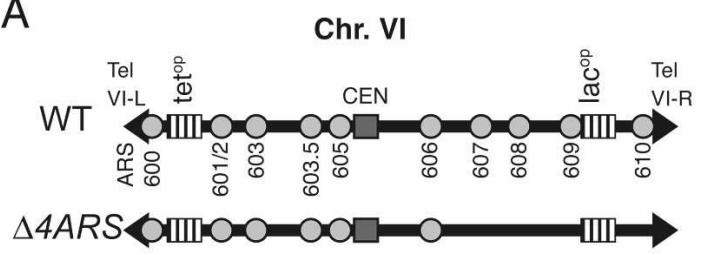

B

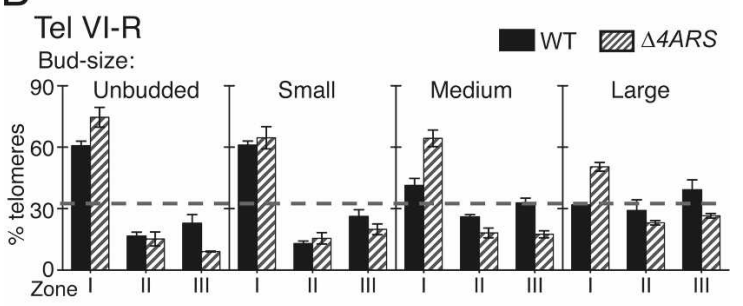

Tel VI-L

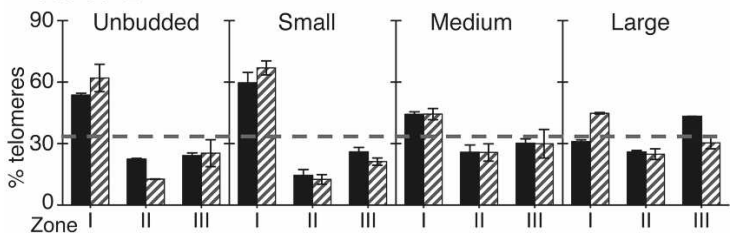

Figure 3. Anchoring of Tel VI-left to the nuclear envelope is independent of the replication status of Tel VI-right. (A) Cartoon illustrating Chr. VI in wild-type (GA-2201) and $\triangle 4 A R S$ (HE95) strains. Tel VI-right is visualized using LacI-CFP fusion proteins binding an inserted array of lac $^{\text {op }}$ repeats. TetR-YFP fusion proteins and an inserted array of tet ${ }^{\text {op }}$ repeats allow visualization of Tel VI-left in the same cells. (B) Positioning of CFP-tagged Tel VI-right and YFP-tagged Tel VI-left were simultaneously observed and quantified in wild-type (Bystricky et al. $2005)$ and $\triangle 4 A R S$ cells. The top graphs shows positioning of Tel VI-right in wild-type (black bars) and $\triangle 4 A R S$ (hatched bars) cells. The bottom graphs show positioning of Tel VI-left observed in the same wild-type and $\triangle 4 A R S$ cells. (For statistics, see Supplemental Table S1.)

$\triangle 4 A R S$ cells (Fig. 3B, bottom graphs, hatched bars). These data therefore confirmed that delocalization of Tel VI-right depends on DNA replication at this telomere, and revealed that the replication time of Tel VI-right does not affect the anchoring status of Tel VI-left.

\section{Telomeres can replicate at the nuclear periphery prior} to dislodgment

Since we had found that telomere delocalization in late interphase directly depends on replication, we wished to investigate the replication-coupled events that cause telomere dislodgment from the nuclear envelope. First, we tested whether the process of replication itself brings telomeres to the nuclear interior. In the nuclei of eukaryotic cells, DNA replication takes place at discrete foci known as replication factories (Meister et al. 2006). The dynamics of replication foci have been studied in live yeast cells, and it appears that DNA polymerases are relatively static so that during replication, the parental
DNA is "reeled in" to the relatively stationary replication factories (Heun et al. 2001a; Kitamura et al. 2006). Therefore, one possible mechanism for replication to cause dislodgment of telomeres would be the formation of replication factories at a distance from the nuclear envelope. Consequently, during replication, telomeres could be pulled away from the periphery as they enter the replication factories. In order to test this possibility, we used time-lapse microscopy to monitor the position of CFP-tagged Tel VI-right in single cells progressing through S phase (Fig. 4A). In addition to telomere location, the intensity of the telomere dot was measured during the time course. Intensity of the CFP-tagged locus approximately doubles following replication, as additional LacI-CFP is recruited to the replicated lac ${ }^{\mathrm{OP}}$ repeats (Kitamura et al. 2006). This previously established method for analyzing DNA replication enabled us to relate the spatial and temporal dynamics of telomere replication in single cells. If replication of Tel VI-right takes place away from the nuclear periphery, the distance between this telomere and the nuclear envelope should increase at the same time or immediately prior to its replication. Alternatively, the telomere may replicate in a replication focus formed at the periphery, with telomeres dislodging from the nuclear envelope after replication. In this case, the intensity of CFP signal from Tel VI-right would increase while the telomere still appears attached to the nuclear envelope.

Representative images from one time course are shown in Figure 4B. Intensity measurements from this particular time course are plotted in the left graph in Figure 4C. Data from three additional cells are shown in Figure 4C (data for all 16 cells are shown in Supplemental Fig. S5). In approximately half of the imaged cells, the telomere remained at the nuclear periphery during replication, while in only a minority of the cells did the telomere clearly replicate away from the periphery. Since telomeres frequently appear to replicate within factories that are at the nuclear periphery, the force that recruits DNA to the replication factory cannot be the sole cause of telomere dislodgment from the nuclear envelope.

In concordance with population-snapshot data presented in Figures 2 and 3, in prereplication time points collectively $(n=102)$, Tel VI-right was significantly enriched at the nuclear periphery (Fig. 4D, "before replication"). However, following replication $(n=122)$, Tel VIright was dislodged from the nuclear periphery in the majority of time points, showing random localization (Fig. 4D, "after replication").

\section{Time-lapse analysis confirms delayed dislodgement of Tel VI-right in $\triangle 4 A R S$ strain}

We monitored replication using a similar time-lapse approach in the $\triangle 4 A R S$ strain (Fig. 3), where replication of Tel VI-right is delayed. Telomere dot intensity, location relative to the nuclear envelope, and bud size were monitored in $16 \triangle 4 A R S$ cells that were progressing through $S$ phase (Supplemental Fig. S6). As expected, Tel VI-right 
replication took place at a significantly larger bud size on average in $\triangle 4 A R S$ than in wild-type cells (Fig. 4E). Using the data from time-lapse analysis (Supplemental Figs. S5, S6) to plot the percentage of small-, medium-, and largebudded cells with Tel VI-right away from the nuclear periphery also revealed a significant delay in telomere dislodgement in the $\triangle 4 A R S$ strain (Fig. 4F). The results of the time-lapse analysis were therefore consistent with those from the "snapshot" analyses shown in Figures 2 and 3 and confirmed that telomere delocalization from the nuclear periphery is linked to DNA replication.

\section{Ku-mediated anchoring of an internal locus} is deactivated by a replication-coupled event

The data presented above show that telomeres dislodge from the nuclear envelope after replication in mediumbudded cells. Telomeres generally remain delocalized as large-budded cells advance toward mitosis (Figs. 2D, 3B). The negative effect of replication on telomere anchoring therefore is not transient, but involves stable down-regulation to prevent reattachment of telomeres after replication. We therefore considered the possibility that telomeric DNA replication leads to stable suppression of one or both of the telomere-anchoring pathways. Ku and Sir4

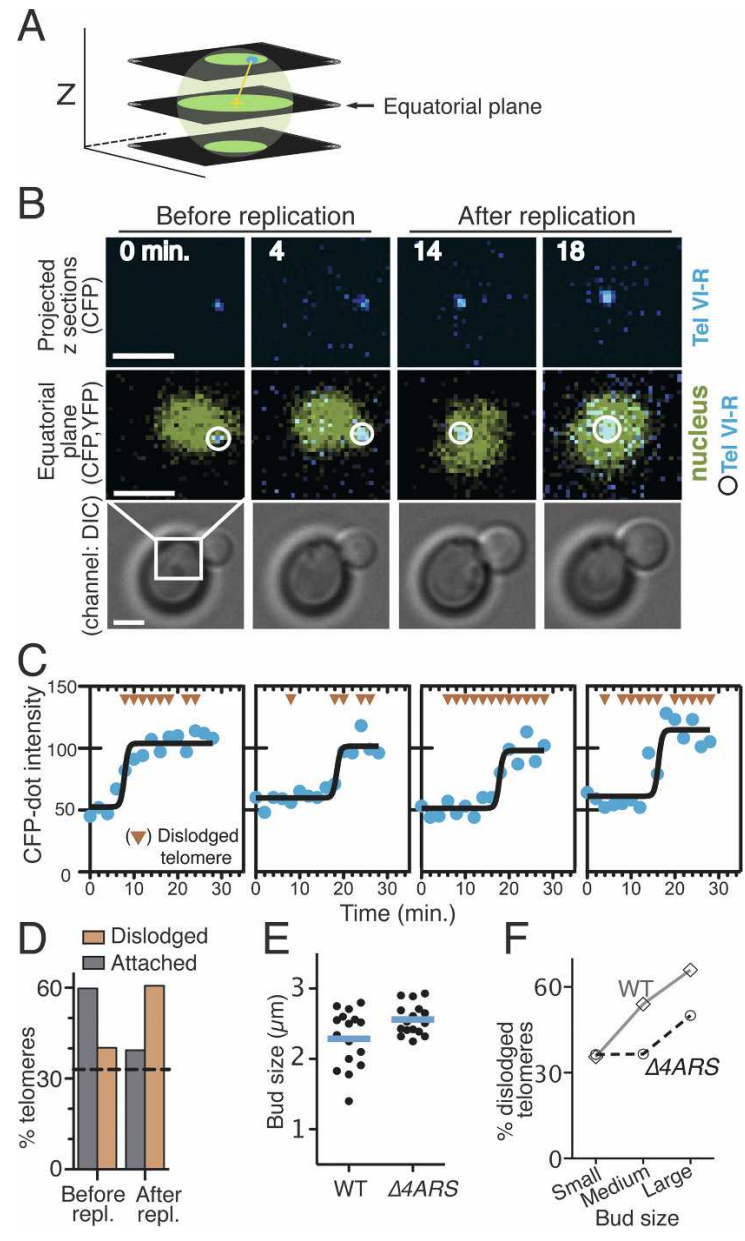

coexist at telomeres and are partially redundant in telomere anchoring (Hediger et al. 2002), so to distinguish any separate effects of replication on the individual pathways, we first used a system in which tethered Ku80 or Sir4 causes artificial peripheral positioning of an internal chromosome locus (Taddei et al. 2004). An internal locus adjacent to ARS607 on chromosome VI (Chr. VI ${ }^{\text {int }}$ ) is normally randomly positioned in the nucleus (Taddei et al. 2004). Chr. VI ${ }^{\text {int }}$ can be targeted to the nuclear periphery by tethering the telomere-anchoring proteins Ku80 or Sir4 to this locus. As shown in Figure 5A, this teth-

Figure 4. Tel VI-right replication can occur at or away from the nuclear periphery, and telomere dislodgement from the nuclear periphery occurs after replication. (A) Illustration of the method used to analyze dot-to-periphery distance. Three Z-stack planes are illustrated; actual images consisted of 16 planes. The center of the nucleus is indicated by + on the equatorial plane. The 3D distance between Tel VI-right (blue circle on top plane) and the center of the nucleus was measured enabling estimation of dotto-periphery distance. $(B)$ Four representative time points from a single time-lapse series. The top panels show the fluorescent CFP signal from Tel VI-right. The middle panels show position of the telomere within the nucleus. In the time points shown, Tel VI-right was on the equatorial plane. The bottom panels show DIC images of the cell when the position and intensity of Tel VI-right were measured. Bars, $2 \mu \mathrm{m} .(C)$ Tel VI-right positioning and intensity data for four representative cells. The intensity of CFP-tagged Tel VI-right (blue circles) is plotted at each imaged time point for four small-budded cells from an asynchronously growing culture of GA-2201 (see data for all 16 cells shown in Supplemental Fig. S5). Images were acquired every $2 \mathrm{~min}$ for $28 \mathrm{~min}$. The inverted red triangles indicate time points at which telomere was detached from the nuclear envelope. To determine the time point at which the telomere is replicating, a curve is fitted over the intensity data points; the midpoint of the intensity increase on the fitted curve is assigned as the replication time. (D) Bar chart showing percent telomere attachment to the nuclear envelope at all time points before $(n=102)$ and after $(n=122)$ replication. The boundary of Zone I in $3 \mathrm{D}$ images of the nuclei is $\sim 110 \mathrm{~nm}$ from the nuclear envelope. This distance is smaller than the resolution of the acquired images (pixel size $\sim 140 \mathrm{~nm}$ in these binned images); hence a telomere in Zone I will be seen as attached. Since Zone I represents one-third of the volume of the nucleus, it is expected that a randomly positioned telomere would be seen as attached in $\sim 33 \%$ of the time points. $P$ values were calculated comparing the observed numbers of attached/detached telomeres with the expected random distributions using a $\chi^{2}$ test (Before repl.: $P=1 \times 10^{-8}$; After repl.: $\left.P=0.21\right)$. $(E)$ Replication of Tel VI-right is delayed in the $\triangle 4 A R S$ strain. The scatter plot shows bud sizes at the time of Tel VI-right replication in wild-type and $\triangle 4 A R S$ (HE95) cells. The horizontal blue line indicates mean bud size (wild type $=2.23 \mu \mathrm{m}, n=16 ; \Delta 4 A R S=2.51 \mu \mathrm{m}, n=16$ ). Timelapse data for $16 \triangle 4 A R S$ cells are shown in Supplemental Figure S6. $(F)$ Percent telomere dislodgment from the periphery in cells observed by time-lapse microscopy for wild type (gray line) and $\triangle 4 A R S$ (blacked dashed line). Time points were categorized according to bud size, and the percent dislodged telomeres was calculated within each bud-size category. Number of time points in each category for wild type: small-budded $=31$, medium-budded $=50$, large-budded $=44$; and for $\triangle 4 A R S$ : smallbudded $=33$, medium-budded $=63$, large-budded $=32$. 


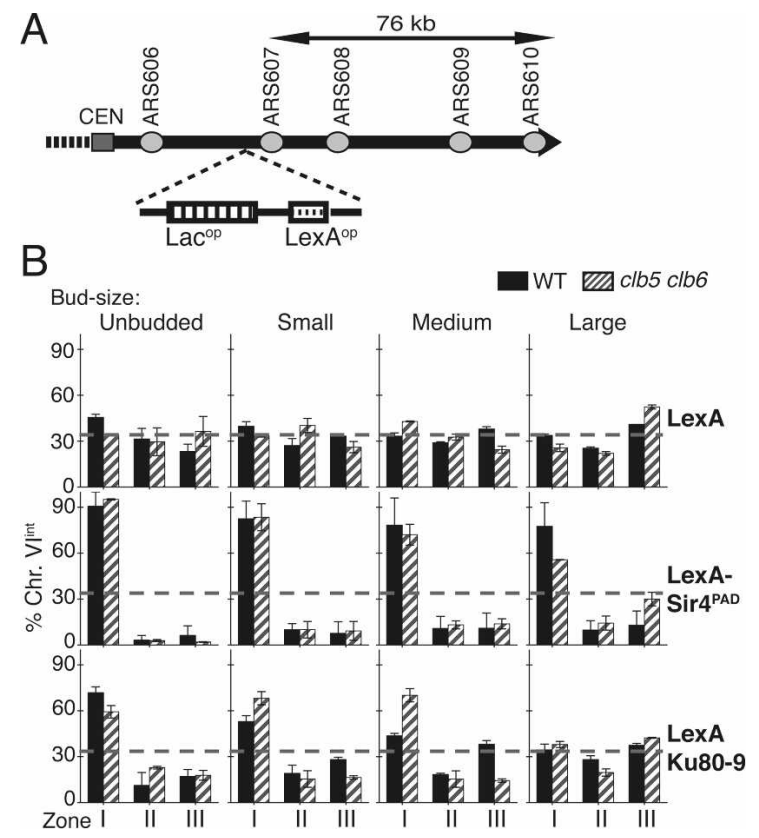

Figure 5. Ku80-mediated anchoring of an internal locus is down-regulated following replication. $(A)$ Schematic cartoon of Chr. VI-R in tethering strain. In addition to lac ${ }^{\mathrm{OP}}$ repeats, an array of LexA-binding sites is inserted adjacent to ARS607 in strain GA-1461 (Taddei et al. 2004). (B) Bar graphs show subnuclear positioning of Chr. VI ${ }^{\text {int }}$ in wild-type (black bars) or clb5 clb6 (hatched bars) cells carrying plasmids expressing LexA, LexA-Ku80-9, or LexA-Sir4 ${ }^{\mathrm{PAD}}$ (top, middle, and bottom graph series, respectively). Expressing LexA alone did not cause relocalization of $\mathrm{Chr}$. VI ${ }^{\text {int }}$. Sir $4^{\mathrm{PAD}}$ tethering was effective throughout the cell cycle in wild-type and clb5 clb6 cells. In wild-type cells, LexA-Ku80-9 tethering was effective only in unbudded and small-budded cells. clb5 clb6 mutants displayed delayed deactivation of Ku80-9 anchoring function. (For significance of chromosome VI ${ }^{\text {int }}$ localization, see Supplemental Table S2.)

ering is achieved by inserting an array of LexA-binding sites $\left(\right.$ LexA $^{\text {op }}$ ) adjacent to ARS607 and expressing the LexAp DNA-binding domain fused to Ku80 or Sir4. Chr. $\mathrm{VI}^{\text {int }}$ is also tagged with GFP by insertion of $1 \mathrm{ac}^{\mathrm{op}}$ repeats, allowing subnuclear positioning of $\mathrm{Chr}$. $\mathrm{VI}^{\text {int }}$ to be examined in live cells. This system has been useful for elucidating the anchoring functions of $\mathrm{Ku}$ and Sir4 proteins (Taddei et al. 2004).

We studied positioning of Chr. VI ${ }^{\text {int }}$ that was localized to the nuclear envelope by LexA-fused Ku80-9 or Sir4 ${ }^{\text {PAD }}$ (Taddei et al. 2004). Ku80-9 and Sir $4^{\mathrm{PAD}}$ are telomeric silencing-incompetent forms of Ku80 and Sir4, which are capable of tethering a randomly positioned locus to the nuclear periphery. As previously reported, expression of LexAp alone did not relocalize Chr. VI ${ }^{\text {int }}$ (Fig. 5B, top graphs, black bars). Sir4 ${ }^{\mathrm{PAD}}$-mediated anchoring was effective throughout the cell cycle, since Chr. VI ${ }^{\text {int }}$ was localized to Zone I in unbudded, small-, medium-, and large-budded cells (Fig. 5B, middle graphs). Expression of the LexA-Ku80-9 fusion resulted in anchoring of Chr. $\mathrm{VI}^{\text {int }}$ at the periphery in unbudded and small-budded cells, but random positioning in medium and large-bud- ded cells (Fig. 5B, bottom graphs). This cell cycle-regulated anchoring effect was strikingly reminiscent of the localization pattern observed for telomeres, suggesting that peripheral tethering by LexA-Ku80-9 may be subject to cell cycle controls similar to those operating on telomeres. Since Ku80-mediated anchoring showed cell cycle delocalization in late interphase, we examined whether replication triggers deactivation of the $\mathrm{Ku}$ anchoring pathway by testing whether delaying DNA replication affects positioning of Chr. VI ${ }^{\text {int }}$. Delaying the onset of S phase by deleting CLB5 and CLB6 extended the localization period of Ku80-9 anchored Chr. VI ${ }^{\text {int }}$, so that it remained significantly enriched in Zone I in medium-budded cells (Fig. 5B, bottom graphs, hatched bars). These data show that down-regulation of the tethering function of Ku80-9 in medium- and large-budded wildtype cells depends on DNA replication. It is noteworthy that this effect is specific to Ku80-9, as the clb5 clb6 mutation did not prolong Sir $4^{\mathrm{PAD}}$ anchoring function. The delocalization of a Ku80-tethered locus in late interphase and the effect of delayed replication on the time of delocalization suggest that replication leads to downregulation of the $\mathrm{Ku}$ tethering function.

\section{Sir-mediated anchoring of Tel XIV-left is not affected by DNA replication}

The LexA tethering system does not mimic all aspects of telomere tethering, so we examined the cell cycle dependence of localization of a natural telomere (XIV-left) under conditions in which it is localized by only the $\mathrm{Ku}$ - or only the Sir4-mediated pathway (Hiraga et al. 2006). In a sir4 background (where only the Ku pathway is available for localization), Tel XIV-left was localized in unbudded and small-budded cells, but became delocalized in medium and large-budded cells (Fig. 6). This result is consistent with replication-linked delocalization of Tel XIVleft due to down-regulation of Ku-mediated tethering. In

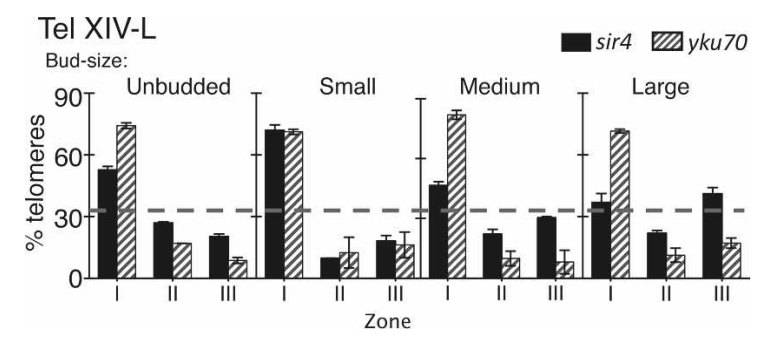

Figure 6. Ku-mediated peripheral positioning of Tel XIV-left is down-regulated in late interphase, but Sir-mediated positioning remains functional. Bar graphs show the localization of Tel XIVleft in sir4 (SHY158) and yku70 mutant cells (SHY155), plotted with notation as in Figure 1. Number of cells observed for SHY158-unbudded: $n_{1}=51, n_{2}=68, P=8.47 \times 10^{-4}$; smallbudded: $n_{1}=71, n_{2}=72, P=3.44 \times 10^{-10} ;$ medium-budded: $n_{1}=57, n_{2}=81, P=1.28 \times 10^{-1}$; large-budded: $n_{1}=63, n_{2}=86$, $P=5.86 \times 10^{-2} ;$ and SHY155-unbudded: $n_{1}=59, n_{2}=41$, $P=5.16 \times 10^{-8}$; small-budded: $n_{1}=70, n_{2}=40, P=4.03 \times 10^{-7}$; medium-budded: $n_{1}=64, n_{2}=44, P=5.47 \times 10^{-12}$; large-budded, $n_{1}=75, n_{2}=51, P=1.28 \times 10^{-8}$. 
a yku70 background, in contrast, Tel XIV-left showed peripheral positioning that was retained even in medium- and large-budded cells (Fig. 6, hatched bars), consistent with the idea that tethering by the Sir4 pathway remains largely unaffected by replication. In this particular experiment, for unknown reasons the localization values were rather higher than previously observed, although the general pattern of localization was similar to previous reports in that the sir4 mutation is more deleterious to localization of Tel XIV-left than is yku70 (Hediger et al. 2002; Hiraga et al. 2006). Taken together, the results in Figures 5 and 6 suggest that telomere delocalization in late interphase is caused by replicationlinked down-regulation of the Ku-mediated peripheral anchoring pathway.

\section{Discussion}

In live yeast cells, anchoring of telomeres to the nuclear envelope is a dynamic process that depends on the $\mathrm{Ku}$ and Sir4 tethering pathways. Studying telomere positioning through the mitotic cell cycle has shown that while telomeres remain localized in the early part of the interphase, they show random subnuclear positioning in late interphase prior to mitosis. Here we used available information on mechanisms of DNA replication to alter replication time of telomeres without affecting other cell cycle events. Delaying telomeric replication postponed telomere dislodgment from the nuclear envelope, showing that dislodgment is dependent on replication. In addition, using single cell time-lapse microscopy, we confirmed that telomere dislodgment takes place in late $S$ phase following replication and showed that telomere replication can take place either at or away from the nuclear periphery.

In order to understand how replication causes dislodgment of telomeres, we examined the effect of replication on individual telomere tethering pathways. Studying the effect of replication on Ku80- and Sir4-mediated artificial tethering showed that the anchoring function of Ku80 is down-regulated following replication (indicated by color change in Fig. 7A). Examining the regulation of Tel XIVleft in sir4 and yku70 mutants confirmed the idea that replication switches off Ku-mediated tethering, but not Sir4-mediated tethering (Fig. 7A). Ku complex remains bound to telomeres following DNA replication (Fisher et al. 2004), and the molecular mechanism responsible for down-regulating $\mathrm{Ku}$-tethering function is not known, but could potentially involve a modification either to Ku80 or to another molecule involved in the pathway (for example, an unidentified attachment component in the nuclear envelope).

At natural telomeres Sir4 and $\mathrm{Ku}$ are both present and their anchoring functions are partially redundant (Hediger et al. 2002). Therefore, while replication may render $\mathrm{Ku}$ incapable of anchoring telomeres, in order to dislodge telomeres the Sir4-mediated anchoring pathway must presumably also be deactivated. Interestingly, it has been shown that Sir4 alone is not sufficient to anchor certain telomeres in cells lacking $\mathrm{Ku}$ function (Hediger et al.
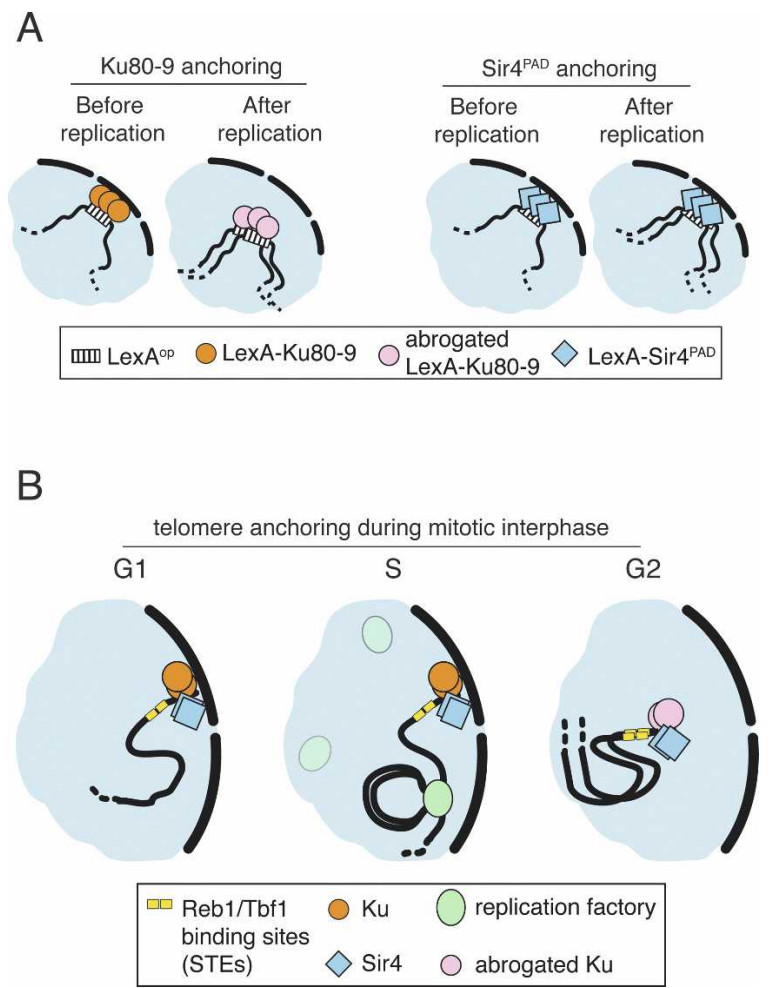

Figure 7. Model for the effects of Ku80 and Sir4 in artificial tethering and natural telomere anchoring before and after replication. (A) Diagram illustrating Ku80-9- and Sir4 ${ }^{\mathrm{PAD}}$-mediated tethering of $\mathrm{Chr} . \mathrm{VI}^{\text {int }}$ to the nuclear periphery. After replication, Ku80-9 anchoring is deactivated; however, $\mathrm{Sir}^{\mathrm{PAD}}$ remains capable of anchoring Chr. VI ${ }^{\text {int }}$ to the nuclear envelope. $(B)$ At native telomeres, $\mathrm{Ku}$ and Sir4 anchoring pathways are partially redundant and during G1 anchor telomeres to the nuclear envelope. During S phase, replication factories are formed, and in late $\mathrm{S}$ phase, the telomeres are replicated. Following telomere replication, Ku-mediated anchoring is switched off. We propose that the antagonizing force of STEs prevents Sir4 from reanchoring telomeres to the nuclear envelope.

2006). However, when a telomere is truncated (by removing its STEs), the Sir4 anchoring pathway becomes sufficient to localize telomeres at the periphery (Hediger et al. 2006). These observations showed that sequence elements present within the subtelomeric regions antagonize the Sir4 anchoring pathway, so that in the absence of these sequence elements Sir4 can tether telomeres to the nuclear envelope. The $S$. cerevisiae STEs consist of the $\mathrm{X}$ core sequence, the subtelomeric repeat elements (STR), and up to four highly conserved repeats known as $\mathrm{Y}^{\prime}$ elements (not present at some telomeres). These elements and their binding factors (e.g., Reb1 and Tbf1) have been demonstrated to antagonize telomere anchoring (Hediger et al. 2006); however, the presence of both $\mathrm{Ku}$ - and Sir-mediated anchoring at telomeres is sufficient to overcome the antagonistic force of the STEs in early interphase. Localization of telomeres therefore may depend on the interplay between telomere-anchoring pathways and factors that antagonize these pathways. Our observations, together with previous results, 
suggest that prior to replication when the anchoring function of $\mathrm{Ku}$ is intact, telomeres are localized at the periphery via the jointly acting Sir4 and $\mathrm{Ku}$ anchoring pathways. However, following replication, after the anchoring function of $\mathrm{Ku}$ is suppressed, the antagonistic force of STEs is greater than the tethering force of Sir4 therefore telomeres become delocalized (Fig. 7B). In this model, the absence of STEs at Chr. VI ${ }^{\text {int }}$ would explain why this artificially tethered locus remains localized by LexA-Sir4 ${ }^{\mathrm{PAD}}$ in medium- and large-budded cells (Fig. 7A, right panel).

If the model described above and shown in Figure 7 is correct, it is perhaps surprising that telomere XIV-left remained peripherally localized in the yku70 background in medium- and large-budded cells (Fig. 6). Tel XIV-left does become delocalized in medium- and largebudded cells in a wild-type background (Fig. 1), which we propose is caused by antagonism of Sir-mediated localization by the Reb1 and Tbf1 STE-binding factors (in conjunction with Ku pathway switch-off). One possible explanation for the differing behavior of Tel XIV-left in wild-type and yku70 backgrounds might be that the yku70 mutation indirectly affects the regulation of the Sir4 localization pathway. For example, a defect in Reb1/ Tbf1 binding to STEs in the yku70 mutant could alleviate the negative effect of STEs on the Sir-mediated positioning pathway, so that the Sir-mediated pathway alone becomes sufficient to position Tel XIV-left in late interphase. $\mathrm{Ku}$ is known to affect telomeric heterochromatin structure (Boulton and Jackson 1998).

The functional significance of the control over telomere localization by replication remains to be determined. The fact that telomeres dislodge from the periphery late in interphase but reattach immediately after nuclear fission (Supplemental Fig. S7) raises the possibility that disengagement is required for correct chromosome segregation. We found that dissociation from the nuclear periphery is not, however, essential for correct mitotic segregation, since a constitutively tethered gene locus is correctly segregated (Supplemental Fig. S8). Extension by telomerase is believed to be coupled to DNA replication, so one interesting possibility is that the association of telomeres with the nuclear periphery must be dissolved for telomerase to access the DNA end. Delaying DNA replication did not substantially affect telomere length (or telomere-proximal transcriptional repression) (Supplemental Fig. S9), but since replication and delocalization do eventually occur in the clb5 clb6 mutant, this observation does not rule out the possibility that delocalization is important for telomerase-mediated end maintenance.

Our results establish a clear link between DNA replication and control of intranuclear chromosome organization. DNA synthesis could potentially have a wider effect on nuclear organization, and yeast cells may rely on chromosome replication to facilitate events that require nuclear reorganization. For instance, it has been shown that replication at centromeric regions causes disassembly of kinetochores and detachment of centromeres from the spindle pole prior to recapture by micro- tubules (Kitamura et al. 2007). In addition, telomere repositioning and bouquet formation during the transition to a meiotic cell cycle is conserved among eukaryotes, and in yeast is shown to be caused by a DNA replicationcoupled event (Trelles-Sticken et al. 2005). Emerging data suggest that mechanisms involved in positioning of telomeres may be conserved from yeast to vertebrates, at least to some extent. For example, the SUN domain proteins Mps3p and SUN2 have been identified as involved in positioning telomeres in yeast and human cells, respectively (Bupp et al. 2007; Conrad et al. 2007; Schmitt et al. 2007). Together these data raise the possibility that DNA replication may contribute to reorganization of higher eukaryotic nuclei during events such as development and cell differentiation.

\section{Materials and methods}

Strains

All strains used were constructed in the W303 background. Strain genotypes and construction methods are indicated in Supplemental Table S3.

\section{Replication timing by dense isotope transfer method}

Dense isotope transfer experiments were carried out based on previously described procedures (McCarroll and Fangman 1988; Donaldson et al. 1998; Hiraga et al. 2006), except that cells were grown at $30^{\circ} \mathrm{C}$ in isotopically dense medium. Subsequently, the cultures were synchronized by $\alpha$-factor and released at $30^{\circ} \mathrm{C}$ in isotopically light medium. Two additional series of samples were taken to analyze budding and DNA content using flow cytometry. Flow cytometry was carried out using SYTOX Green nucleic acid stain (Invitrogen Molecular Probes) as previously described (Haase and Reed 2002).

\section{Microscopy and image analysis}

The procedure for preparing live-cell samples for snapshot imaging (Figs. 1-3, 5, 6) was described by Hiraga et al. (2006). An Applied Precision Deltavision RT microscope system was used to acquire images using a $100 \times$ objective (NA 1.4) without binning. Cross-sections of the cells were captured in Z-stacks of 21 images, with a distance of $0.25 \mu \mathrm{m}$ between each Z-plane. Applied Precision softWorX Explorer was used to analyze dot-toperiphery distance, nuclear diameter, and bud size. The approach for analyzing positioning of the telomere dot within the nuclear zones was described previously (Hediger et al. 2002). Zones I, II, and III have equal area, hence each zone occupies $33 \%$ of the total area within the nuclear rim. A randomly positioned locus is therefore expected to be positioned in each zone with equal frequency $(33 \%)$. Consistently, internal loci that are not tethered to the nuclear envelope typically give values close to $33 \%$ in this assay (e.g., untethered Chr. VI int in Fig. $5)$. To ensure integrity of data, the identities of some strains were concealed from the experimenter during imaging and analysis.

For time-lapse microscopy (Fig. 4), the cells were grown in SCD + YPD (3:1) medium and mounted in a mini-Petri dish coated with Concanavalin A. To prevent rapid bleaching, a 10\% ND filter was used. During time-lapse acquisition, sensitivity of the CCD camera was increased by $2 \times 2$ binning of pixels. The images were analyzed using AppliedPrecision softWorx Ex- 
plorer. The 3D position of the telomere dot in the nucleus was determined in Z-stack images before creating maximal projections of each image to measure telomere dot intensity. Plotting and One Site-LogIC50 analysis of intensity data were carried out as described previously (Kitamura et al. 2006).

\section{Acknowledgments}

Thanks to all members of the Donaldson laboratory for comments and technical advice, and in particular to Shin-ichiro Hiraga for advice on microscopy techniques. We thank Susan Gasser for providing strains and constructs. David Gilbert and Junjie Lu (both of Florida State University) and David Lydall (Newcastle University) provided valuable comments on the manuscript. H.E. was funded by ORSAS Scotland and a University of Aberdeen 6th Century Scholarship. This work was partly funded by Wellcome grant $082377 / \mathrm{Z} / 07 / \mathrm{Z}$.

\section{References}

Andrulis, E.D., Neiman, A.M., Zappulla, D.C., and Sternglanz, R. 1998. Perinuclear localization of chromatin facilitates transcriptional silencing. Nature 394: 592-595.

Aparicio, O.M., Billington, B.L., and Gottschling, D.E. 1991. Modifiers of position effect are shared between telomeric and silent mating-type loci in S. cerevisiae. Cell 66: 12791287.

Boulton, S.J. and Jackson, S.P. 1998. Components of the kudependent non-homologous end-joining pathway are involved in telomeric length maintenance and telomeric silencing. $E M B O$ I. 17: 1819-1828.

Brickner, J.H. and Walter, P. 2004. Gene recruitment of the activated INO1 locus to the nuclear membrane. PLOS Biol. 2: e342. doi: 10.1371/journal.pbio.0020342.

Brickner, D.G., Cajigas, I., Fondufe-Mittendorf, Y., Ahmed, S., Lee, P.C., Widom, J., and Brickner, J.H. 2007. H2A.Z-mediated localization of genes at the nuclear periphery confers epigenetic memory of previous transcriptional state. PLoS Biol. 5: e81. doi: 10.1371/journal.pbio.0050081.

Bupp, J.M., Martin, A.E., Stensrud, E.S., and Jaspersen, S.L. 2007. Telomere anchoring at the nuclear periphery requires the budding yeast Sad1-UNC-84 domain protein Mps3. I. Cell Biol. 179: 845-854.

Bystricky, K., Laroche, T., van Houwe, G., Blaszczyk, M., and Gasser, S.M. 2005. Chromosome looping in yeast: Telomere pairing and coordinated movement reflect anchoring efficiency and territorial organization. J. Cell Biol. 168: 375387.

Casolari, J.M., Brown, C.R., Drubin, D.A., Rando, O.J., and Silver, P.A. 2005. Developmentally induced changes in transcriptional program alter spatial organization across chromosomes. Genes \& Dev. 19: 1188-1198.

Cockell, M. and Gasser, S.M. 1999. Nuclear compartments and gene regulation. Curr. Opin. Genet. Dev. 9: 199-205.

Conrad, M.N., Lee, C.Y., Wilkerson, J.L., and Dresser, M.E. 2007. MPS3 mediates meiotic bouquet formation in Saccharomyces cerevisiae. Proc. Natl. Acad. Sci. 104: 8863-8868.

Cremer, T. and Cremer, C. 2001. Chromosome territories, nuclear architecture and gene regulation in mammalian cells. Nat. Rev. Genet. 2: 292-301.

Donaldson, A.D., Raghuraman, M.K., Friedman, K.L., Cross, F.R., Brewer, B.J., and Fangman, W.L. 1998. CLB5-dependent activation of late replication origins in S. cerevisiae. Mol. Cell 2: 173-182.

Epstein, C.B. and Cross, F.R. 1992. CLB5: A novel B cyclin from budding yeast with a role in S phase. Genes \& Dev. 6: 16951706.

Ferguson, B.M., Brewer, B.J., Reynolds, A.E., and Fangman, W.L. 1991. A yeast origin of replication is activated late in $S$ phase. Cell 65: 507-515.

Fisher, T.S. and Zakian, V.A. 2005. Ku: A multifunctional protein involved in telomere maintenance. DNA Repair (Amst.) 4: 1215-1226.

Fisher, T.S., Taggart, A.K. and Zakian, V.A. 2004. Cell cycledependent regulation of yeast telomerase by ku. Nat. Struct. Mol. Biol. 11: 1198-1205.

Friedman, K.L., Diller, J.D., Ferguson, B.M., Nyland, S.V., Brewer, B.J., and Fangman, W.L. 1996. Multiple determinants controlling activation of yeast replication origins late in S phase. Genes \& Dev. 10: 1595-1607.

Friedman, K.L., Brewer, B.J., and Fangman, W.L. 1997. Replication profile of Saccharomyces cerevisiae chromosome VI. Genes Cells 2: 667-678.

Funabiki, H., Hagan, I., Uzawa, S., and Yanagida, M. 1993. Cell cycle-dependent specific positioning and clustering of centromeres and telomeres in fission yeast. J. Cell Biol. 121: 961-976.

Gotta, M., Laroche, T., Formenton, A., Maillet, L., Scherthan, H., and Gasser, S.M. 1996. The clustering of telomeres and colocalization with Rap1, Sir3, and Sir4 proteins in wild-type Saccharomyces cerevisiae. J. Cell Biol. 134: 1349-1363.

Gottschling, D.E., Aparicio, O.M., Billington, B.L., and Zakian, V.A. 1990. Position effect at $S$. cerevisiae telomeres: Reversible repression of pol II transcription. Cell 63: 751-762.

Haase, S.B. and Reed, S.I. 2002. Improved flow cytometric analysis of the budding yeast cell cycle. Cell Cycle 1: 132-136.

Hayashi, A., Ogawa, H., Kohno, K., Gasser, S.M., and Hiraoka, Y. 1998. Meiotic behaviours of chromosomes and microtubules in budding yeast: Relocalization of centromeres and telomeres during meiotic prophase. Genes Cells 3: 587-601.

Hediger, F., Neumann, F.R., Van Houwe, G., Dubrana, K., and Gasser, S.M. 2002. Live imaging of telomeres: YKu and sir proteins define redundant telomere-anchoring pathways in yeast. Curr. Biol. 12: 2076-2089.

Hediger, F., Taddei, A., Neumann, F.R., and Gasser, S.M. 2004. Methods for visualizing chromatin dynamics in living yeast. Methods Enzymol. 375: 345-365.

Hediger, F., Berthiau, A.S., van Houwe, G., Gilson, E., and Gasser, S.M. 2006. Subtelomeric factors antagonize telomere anchoring and Tell-independent telomere length regulation. EMBO T. 25: 857-867.

Heun, P., Laroche, T., Raghuraman, M.K., and Gasser, S.M. 2001a. The positioning and dynamics of origins of replication in the budding yeast nucleus. J. Cell Biol. 152: 385-400.

Heun, P., Laroche, T., Shimada, K., Furrer, P., and Gasser, S.M. 2001b. Chromosome dynamics in the yeast interphase nucleus. Science 294: 2181-2186.

Hiraga, S., Robertson, E.D., and Donaldson, A.D. 2006. The Ctf18 RFC-like complex positions yeast telomeres but does not specify their replication time. EMBO J. 25: 1505-1514.

Ishii, K., Arib, G., Lin, C., Van Houwe, G., and Laemmli, U.K. 2002. Chromatin boundaries in budding yeast: The nuclear pore connection. Cell 109: 551-562.

Kitamura, E., Blow, J.J., and Tanaka, T.U. 2006. Live-cell imaging reveals replication of individual replicons in eukaryotic replication factories. Cell 125: 1297-1308.

Kitamura, E., Tanaka, K., Kitamura, Y., and Tanaka, T.U. 2007. Kinetochore microtubule interaction during $S$ phase in Saccharomyces cerevisiae. Genes \& Dev. 21: 3319-3330.

Kosak, S.T., Skok, J.A., Medina, K.L., Riblet, R., Le Beau, M.M., Fisher, A.G., and Singh, H. 2002. Subnuclear compartmen- 
talization of immunoglobulin loci during lymphocyte development. Science 296: 158-162.

Kuhne, C. and Linder, P. 1993. A new pair of B-type cyclins from Saccharomyces cerevisiae that function early in the cell cycle. $E M B O$ J. 12: 3437-3447.

Kumaran, R.I., Thakar, R., and Spector, D.L. 2008. Chromatin dynamics and gene positioning. Cell 132: 929-934.

Laroche, T., Martin, S.G., Gotta, M., Gorham, H.C., Pryde, F.E., Louis, E.J., and Gasser, S.M. 1998. Mutation of yeast ku genes disrupts the subnuclear organization of telomeres. Curr. Biol. 8: 653-656.

Laroche, T., Martin, S.G., Tsai-Pflugfelder, M., and Gasser, S.M. 2000. The dynamics of yeast telomeres and silencing proteins through the cell cycle. J. Struct. Biol. 129: 159-174.

Louis, E.J. and Vershinin, A.V. 2005. Chromosome ends: Different sequences may provide conserved functions. Bioessays 27: 685-697.

Maillet, L., Boscheron, C., Gotta, M., Marcand, S., Gilson, E., and Gasser, S.M. 1996. Evidence for silencing compartments within the yeast nucleus: A role for telomere proximity and sir protein concentration in silencer-mediated repression. Genes \& Dev. 10: 1796-1811.

McCarroll, R.M. and Fangman, W.L. 1988. Time of replication of yeast centromeres and telomeres. Cell 54: 505-513.

Meaburn, K.J. and Misteli, T. 2008. Locus-specific and activityindependent gene repositioning during early tumorigenesis. J. Cell Biol. 180: 39-50.

Meister, P., Taddei, A., and Gasser, S.M. 2006. In and out of the replication factory. Cell 125: 1233-1235.

Palladino, F., Laroche, T., Gilson, E., Axelrod, A., Pillus, L., and Gasser, S.M. 1993. SIR3 and SIR4 proteins are required for the positioning and integrity of yeast telomeres. Cell 75: 543-555.

Postberg, J., Juranek, S.A., Feiler, S., Kortwig, H., Jonsson, F., and Lipps, H.J. 2001. Association of the telomere-telomerebinding protein complex of hypotrichous ciliates with the nuclear matrix and dissociation during replication. J. Cell Sci. 114: 1861-1866.

Raghuraman, M.K., Winzeler, E.A., Collingwood, D., Hunt, S., Wodicka, L., Conway, A., Lockhart, D.J., Davis, R.W., Brewer, B.J., and Fangman, W.L. 2001. Replication dynamics of the yeast genome. Science 294: 115-121.

Robinett, C.C., Straight, A., Li, G., Willhelm, C., Sudlow, G., Murray, A., and Belmont, A.S. 1996. In vivo localization of DNA sequences and visualization of large-scale chromatin organization using lac operator/repressor recognition. J. Cell Biol. 135: 1685-1700.

Schmitt, J., Benavente, R., Hodzic, D., Hoog, C., Stewart, C.L., and Alsheimer, M. 2007. Transmembrane protein Sun2 is involved in tethering mammalian meiotic telomeres to the nuclear envelope. Proc. Natl. Acad. Sci. 104: 7426-7431.

Schwob, E. and Nasmyth, K. 1993. CLB5 and CLB6, a new pair of B cyclins involved in DNA replication in Saccharomyces cerevisiae. Genes \& Dev. 7: 1160-1175.

Taddei, A. and Gasser, S.M. 2004. Multiple pathways for telomere tethering: Functional implications of subnuclear position for heterochromatin formation. Biochim. Biophys. Acta 1677: 120-128.

Taddei, A., Hediger, F., Neumann, F.R., Bauer, C., and Gasser, S.M. 2004. Separation of silencing from perinuclear anchoring functions in yeast Ku80, Sir4 and Esc1 proteins. EMBO J. 23: 1301-1312.

Taddei, A., Van Houwe, G., Hediger, F., Kalck, V., Cubizolles, F., Schober, H., and Gasser, S.M. 2006. Nuclear pore association confers optimal expression levels for an inducible yeast gene. Nature 441: 774-778.
Therizols, P., Fairhead, C., Cabal, G.G., Genovesio, A., OlivoMarin, J.C., Dujon, B., and Fabre, E. 2006. Telomere tethering at the nuclear periphery is essential for efficient DNA double strand break repair in subtelomeric region. I. Cell Biol. 172: 189-199.

Trelles-Sticken, E., Bonfils, S., Sollier, J., Geli, V., Scherthan, H., and de La Roche Saint-Andre, C. 2005. Set1- and Clb5-deficiencies disclose the differential regulation of centromere and telomere dynamics in Saccharomyces cerevisiae meiosis. J. Cell Sci. 118: 4985-4994.

Yamashita, M., Hori, Y., Shinomiya, T., Obuse, C., Tsurimoto, T., Yoshikawa, H., and Shirahige, K. 1997. The efficiency and timing of initiation of replication of multiple replicons of Saccharomyces cerevisiae chromosome VI. Genes Cells 2: 655-665. 


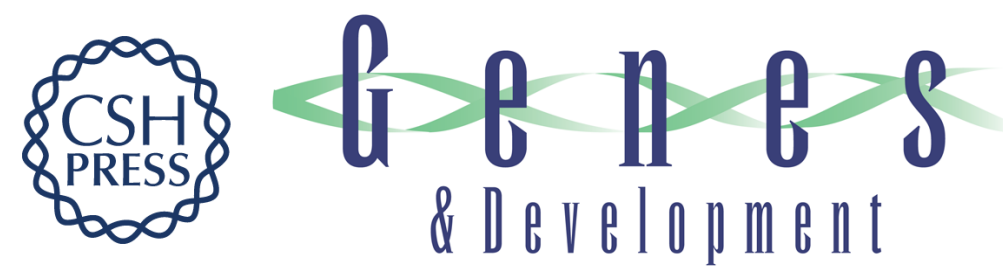

\section{Release of yeast telomeres from the nuclear periphery is triggered by replication and maintained by suppression of Ku-mediated anchoring}

Hani Ebrahimi and Anne D. Donaldson

Genes Dev. 2008, 22:

Access the most recent version at doi:10.1101/gad.486208

Supplemental http://genesdev.cshlp.org/content/suppl/2008/11/18/22.23.3363.DC1
Material

References This article cites 54 articles, 23 of which can be accessed free at:

http://genesdev.cshlp.org/content/22/23/3363.full.html\#ref-list-1

License

Email Alerting Receive free email alerts when new articles cite this article - sign up in the box at the top

Service

right corner of the article or click here.

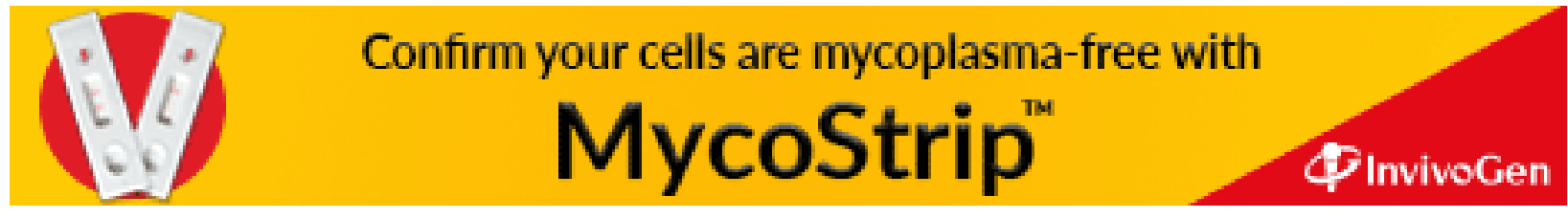

